\title{
Prediction of Mechanical properties of human rib cortical bone using fractal dimension
}

\author{
J. Velázquez-Ameijide ${ }^{a *}$ S. García-Vilana ${ }^{a}$, D. Sánchez-Molina ${ }^{a}$, \\ J.Llumà ${ }^{b}$, E. Martínez-González ${ }^{a}$, M.Carmen Rebollo-Soria ${ }^{c}$, \\ C. Arregui-Dalmases ${ }^{d}$,
}

11 October, 2020

${ }^{a}$ UPC, EEBE-GRABI, Barcelona, Spain

${ }^{b}$ UPC, EEBE-PROCOMAME, Barcelona, Spain

${ }^{c}$ IMLA-Subdirección de Huesca, Huesca, Spain

${ }^{e}$ Centro Zaragoza, Pedrola, Spain

Juan Velázquez-Ameijide, Silvia García-Vilana, David Sánchez-Molina, Jordi Llumà , Eva Martínez-González , M. Carmen Rebollo-Soria \& Carlos Arregui-Dalmases (2020): Prediction of mechanical properties of human rib cortical bone using fractal dimension, Computer Methods in Biomechanics and Biomedical Engineering, DOI: 10.1080/10255842.2020.1836623

\begin{abstract}
A large number of post mortem human subjects was used to investigate the relation between the micro-structure of rib cortical bone and the mechanical properties using Fractal Dimension. Uniaxial tensile tests were performed on coupons of rib cortical bone. Tensile strength, yield stress, Young's Modulus, maximum strain, and work to fracture were determined for each cou- pon. Fractal dimension was computed using CT images and Digital Image Correlation proce- dures. A highly significant effect of fractal dimension in the mechanical properties was found. In addition, the variation in mechanical properties was found to be adequately represented by Generalized Extreme Value type distributions.

KEYWORDS: Medical Imaging, Human Rib, Fractal Dimension, Cortical Bone, Mechanical Properties.
\end{abstract}

\section{Introduction}

Mechanical properties of bone have been extensively studied in the past decades for different purposes. Among them is the prediction of bone mechanical failure which can be used, for instance, to develop reliable Finite Element Models

${ }^{*}$ Corresponding author: juan.velazquez@upc.edu 
(FEM) for restraint systems in vehicles. Typically, the mechanical properties cannot be determined in vivo [Töyräs et al., 1999]. However, the use of descriptors of bone micro-structure (density, porosity, and fractal dimension) enables one to predict mechanical properties and bone strength, considering that bone internal micro-structure has been identified as a major factor influencing its mechanical properties, in particular its densitometry [Sánchez \& Uzcátegui, 2011]. Different parameters have been defined to characterize bone micro-structure. For example, apparent density and Broadband Ultrasound Attenuation (BUA) were described as structural and compositional predictors for the mechanical properties of cancellous bone. Although BUA exhibits a good correlation with Young's modulus, BUA does not seem to be susceptible enough to small variations [Rho et al., 1997].

Other techniques such as Dual X-ray Absorptiometry (DXA) [Lin et al., 1999], ultrasound techniques, or Speed of Sound (SoS) were also used to describe properties such as the areal Bone Mineral Density (BMD) or the stiffness in cancellous bone. The two previously mentioned magnitudes are sensitive to the bone thickness and the specimen size [Töyräs et al., 1999]. This fact affects directly the values of the properties obtained with these techniques, depending on the tissue or bone analyzed. Moreover, being the cancellous bone the tissue with higher porosity, those techniques may not be appropriate to study cortical bone structure.

Although the influence of the micro-structure is a relevant factor, some studies showed that BMD alone is not enough to describe the presence of pathologies like osteoporosis. This happens because in osteoporosis and other pathologies, the internal bone quality also plays an important role and BMD is a scalar quantity, which does not explicitly characterize the micro-structure or the density distribution [Harrar \& Jennane, 2015]. Due to this insufficiency of BMD, other parameters such as the fabric were intended to characterize the missing factors. Fabric has been used to describe the structure of cancellous bone but, despite showing a good correlation with the elastic properties of cancellous bone [Odgaard et al., 1997], it was observed that fabric only described the alignment in the bone micro-structure [Haire et al., 1998]. Nevertheless, fabric or BMD does not capture some aspects that are well represented by Fractal Dimension (FD). FD is a geometric parameter that describes the complexity of the micro-structure and conveys information about the spatial organization of bone [Haire et al., 1998, Dougherty \& Henebry, 2001].

More precisely, FD is a non-dimensional metric parameter which measures the irregularity of a geometrical structure at different scales (it is especially useful when a number of microscopic details are observed only at small scales). An interesting practical aspect of FD is that it can be determined by means of Digital Image Correlation (DIC) procedures, just on the basis of Computer Tomography (CT) images and at small scales, CT's of cortical bone evidenced large differences in density distribution. These changes can be adequately represented by FD, even when deterministic fractals (see Figure 1) seem much more regular than bone micro-structure [Sánchez-Molina et al., 2013]. Indeed, 
the bone micro-structure at different scales can be represented by a statistical self-similar set in three dimensions (X-Y-grey scale) which FD encodes. Then, FD represents geometrical properties of this self-similar set; thus, the irregularities in density distribution and major geometrical characteristics of bone micro-structure can be adequately represented by this parameter.

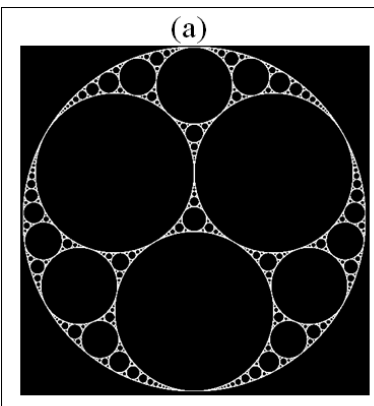

$F D \sim 1.33$

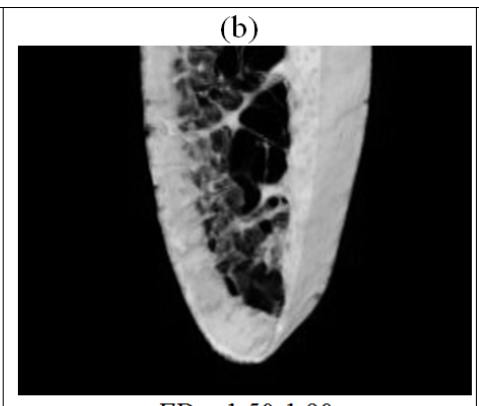

$F D \sim 1.50-1.90$

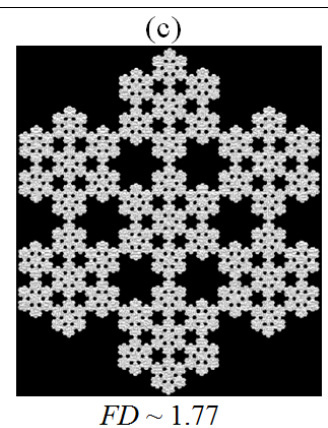

$F D \sim 1.77$

Figure 1: Visual comparison of the micro-structure of cortical bone with two deterministic fractals: (a) Apollonian gasket (FD 1.33) is too sparse to represent cortical bone, (b) real cortical bone (color adjusted, FD is calculated using just cortical bone part), the high-density and low-density parts are intermixed in a complex fractal pattern, and (c) Hexaflake (FD 1.77), on average, has a similar FD of the cortical bone (two main differences are that cortical bone is not deterministic and has non-homogeneous density). In addition, the resemblance between fractal objects of the same FD depends on visual lacunarity (cortical bone is much less 'lacunar').

In fact, FD is a numerical value providing a statistical measure of complexity or roughness, comparing the space-filling capacity of a pattern or material at different scales. Thus, FD gives an idea of the irregularity of distributed properties. In this study, we use FD in order to characterize the variation in density of cortical bone related to BMD and mechanical properties.

The FD has been used in other studies of bone [Haire et al., 1998, Lespessailles et al., 2008, Kerrigan et al., 2014, Harrar et al., 2015], showing that the higher the presence of regions of low density in cortical bone, the higher the FD. It has been reported that FD is not only a better predictor of strength than Singh index [Lin et al., 1999], but in addition, it correlates better with Young's modulus [Rho et al., 1997] than other parameters such as the fabric or the porosity [Haire et al., 1998]. Some authors have suggested that FD is complementary to BMD, due to its mutual low correlation, indicating that FD describes additional independent information [Lin et al., 1999], so both magnitudes together can better predict the mechanical properties. In addition, FD can also distinguish between osteoporotic and healthy bones [Harrar \& Jennane, 2015], showing a good correlation with bone density [Lespessailles et al., 1998].

However, few research has been specifically centered in the study of FD of compact bone and its influence on mechanical properties [Zioupos et al., 2006, Sánchez-Molina et al., 2013, Kerrigan et al., 2014]. Basically, one of these studies focused on the relation of mechanical properties and FD from 10 specimens 
of 6th and 7th rib levels, finding a negative correlation of Young's modulus and FD [Sánchez-Molina et al., 2013]. This article shows a clear relationship of the mechanical properties with the FD, measured in a large number $n=66$ of tensile tests of human rib cortical bone tissue specimens. In addition, given the size of the sample this study enables us to find the distributions of the mechanical properties for general population (ages ranged from 21 to 99 years).

\section{Data and methods}

A sample of human cortical bone ribs were harvested from the anterior and lateral regions of human ribs and a large number of coupons $(n=66)$ were machined from the ribs of the sample. This section summarizes the origin of specimens of the sample, the preparation of these specimens, the tensile testing setting and the data processing of the test data. The influence of FD on the mechanical properties of the ribs has been examined and, in addition, the effect of the individual's anthropometric variables also has been investigated.

\subsection{Materials, preparation, and tensile tests}

The materials used in this study consisted of fresh human rib specimens, harvested from forensic autopsies conducted at the Forensic Pathology Service of the Legal Medicine and Forensic Science Institute of Catalonia (IMLCFC). All the specimens were initially removed for complementary medical-legal investigation. This study was approved by the Research and Ethics committee of the IMLCFC. All the specimens were obtained from the 7th rib of 51 deceased subjects, 34 males and 17 females (from some individuals, multiple samples were obtained). The subjects ages $55.3 \pm 20.1$ years on average (ages ranged from 21 to 91 years), and the average Body Mass Index (BMI) was $29.2 \pm 5.7 \mathrm{~kg} / \mathrm{m}^{2}$ (from 21.2 to $43.8 \mathrm{~kg} / \mathrm{m}^{2}$ ).

Bone coupons were machined from the ribs following the method described in [Sánchez-Molina et al., 2013] (see Figure 2). In this procedure, firstly, soft tissue of the ribs was removed using a scalpel. Secondly, the ribs were divided in different short segments, and from each of these sections, a cortical bone slice was obtained using a SYJ-160 low speed diamond saw, with saline solution as coolant (Figure 2(a-d)).

Thirdly, in each slice two holes of $2 \mathrm{~mm}$ diameter were drilled (Figure 2(e)) and the bone slices were machined with a milling machine, to obtain the desired coupon dog-shape geometry (Figure 2(f)), where the width of each coupon was $2 \mathrm{~mm}$. Fourthly, each coupon was polished to ensure a uniform thickness, verified with a micrometer (Figure $2(\mathrm{~g})$ ). This measurement was important to accurately determine the area of the cross section (Figure $2(\mathrm{~h})$ ).

Finally, coupons were kept hydrated in saline solution prior to the mechanical test to ensure its preservation. The tensile test method in this study was previously used, and described in the literature [Sánchez-Molina et al., 2013]. Tensile tests were performed using a MicroTEST ${ }^{\circledR}$ EM2/20 (MicroTEST, Madrid). 

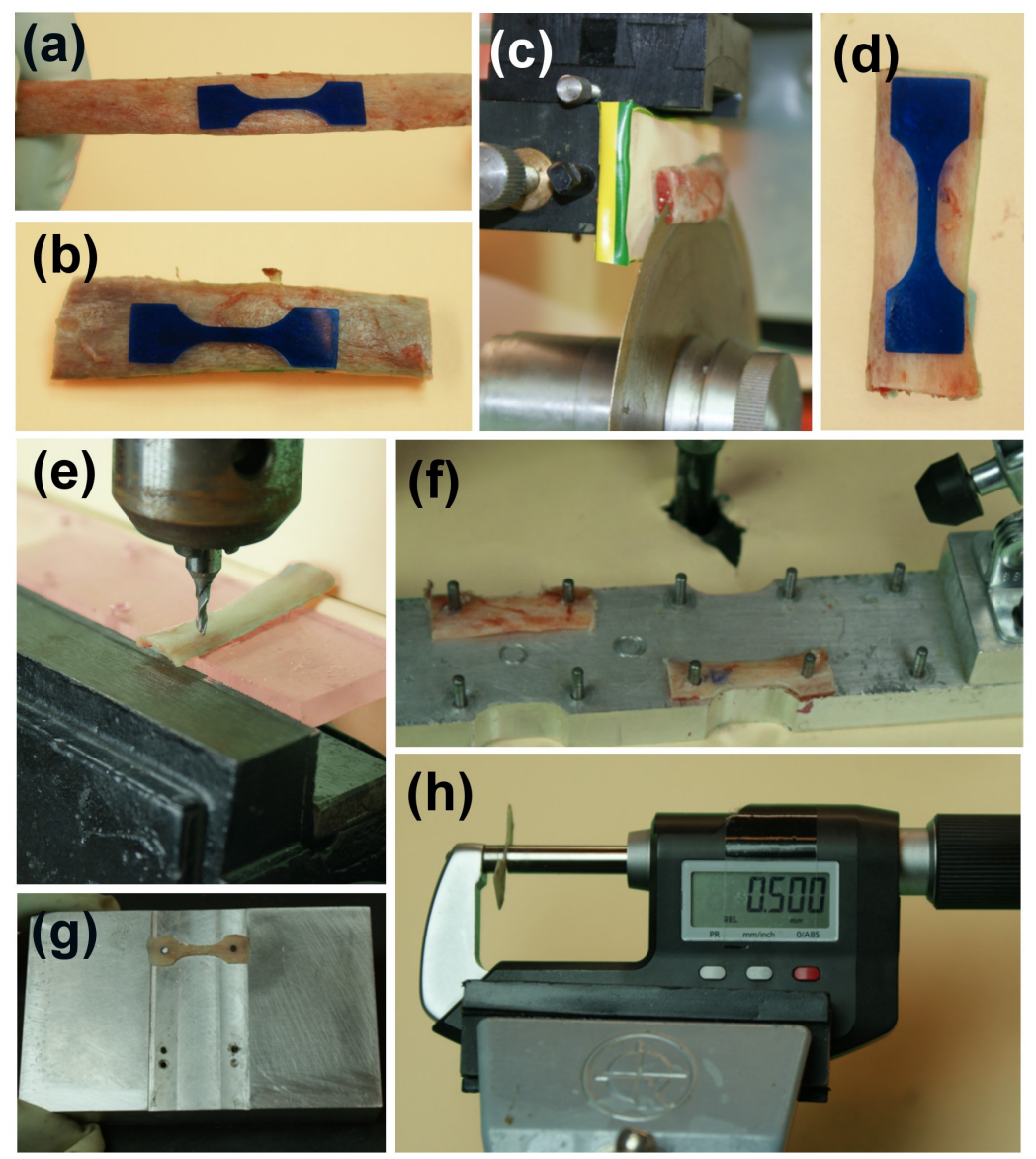

Figure 2: Manufacturing process of coupons from cortical bone: location (a), rib segment (b), sawing (c), cortical slicing (d), drilling (e), machining (f), polishing (g), and gauge length measurement $(\mathrm{h})$.

The coupons were held by means of two special clamping jaws. The load was measured with a load cell of $500 \mathrm{~N}$ coupled to a data acquisition system Spider 8-30 from HBM (Spider, Darmstadt). The strain was obtained from a video of the tensile test, which was recorded with a high-speed camera and analyzed by DIC techniques. The video was processed with a DIC motion tracking software to obtain the strain value of each moment of the test (Section 2.3). In order to determine the coupon elongation in the video, coupons were painted with a random pattern of black dots. Specimens were tested at a strain rate of $\dot{\varepsilon}=0.0138$ $\mathrm{s}^{-1}$ to ensure quasi-static conditions and enough data acquisition before fracture. 


\section{$2.2 \quad$ Fractal dimension}

In this subsection, the measurement of FD is explained in detail and the relation of FD with the mechanical properties (Young's modulus and tensile strength) is clarified. In practice, some procedures are used to compute FD: fractal signature, box counting [Sindeaux et al., 2014, Yin et al., 2015] or power spectrum of Fast Fourier Transform (FFT) [Dougherty and Henebry, 2001, Sánchez-Molina et al., 2013].

For this study, a numerical approximation of Hausdorff-Besicovitch fractal dimension was used. The numerical procedure involves computing the FFT for different images of cross sections of cortical bone specimens. The cross-sectional images are obtained from a CT scanning of each cortical bone coupon specimen. For each digital CT image of a cross section, the FFT was computed and its radially-averaged power spectrum $S(\omega)$ was calculated (see below). Then, the well-known asymptotic behavior of power spectrum is used to obtain explicitly a value for $\mathrm{FD}$, as described below.

Firstly, note that a gray image can be conceived as a gray-scale function $G_{s}(x, y)$, where $(x, y)$ are the coordinates of the pixel and $G_{s}$ the level of gray. The Fourier transform of this function describing the image is given by the following:

$$
\hat{G}_{s}\left(\omega_{x}, \omega_{y}\right)=\int_{-\infty}^{\infty} \int_{-\infty}^{\infty} G_{s}(x, y) e^{-2 \pi i\left(\omega_{x} x+\omega_{y} y\right)} \mathrm{d} x \mathrm{~d} y
$$

$G_{s}(x, y)=0$ when $x>x_{\max }$ or $y>y_{\max }$, so only the region occupied by the image contributes to the above integral. This Fourier Transform is computed numerically using a FFT procedure. After the numerical computation of the integral, we made a change of variables $\left(\omega_{x}, \omega_{y}\right) \mapsto(\omega, \alpha)$ by using the complex relation $\omega e^{i \alpha}=\omega_{x}+i \omega_{y}$, so we define the function $\tilde{G}_{s}(\omega, \alpha)$ by means of the equation $\tilde{G}_{s}(\omega, \alpha)=\hat{G}_{s}\left(\omega_{x}, \omega_{y}\right)=\hat{G}_{s}(\omega \cos \alpha, \omega \sin \alpha)$.

Secondly, once the function has been calculated, we define the radially-averaged power spectrum $S(\omega)$ explicitly:

$$
S(\omega)=\frac{1}{2 \pi} \int_{0}^{2 \pi}\left|\tilde{G}_{s}(\omega, \alpha) \tilde{G}_{s}^{*}(\omega, \alpha)\right| \mathrm{d} \alpha
$$

After computing the radially-averaged power spectrum $S(\omega)$, it can be seen in figure 3, that for high frequencies $\omega$ (equivalently, for low wavelengths), it decays asymptotically as $S(\omega) \sim a \omega^{-\alpha}$. Therefore, the fractal dimension can be computed as FD $=4-\alpha / 2$ [Dougherty \& Henebry, 2001].

For each coupon, five different images of cross sections were considered, and for each cross-sectional image, the radially-averaged power spectrum $S(\omega)$ was computed. Each cross section provides a value for FD. The average of these values of $\mathrm{FD}$ is computed and is the reference value for each coupon. All the above computations can be made numerically using FFT and DIC procedures (all the key assumptions, in the previous derivations, are justified by the theoretical body known as fractal geometry or fractal analysis). 


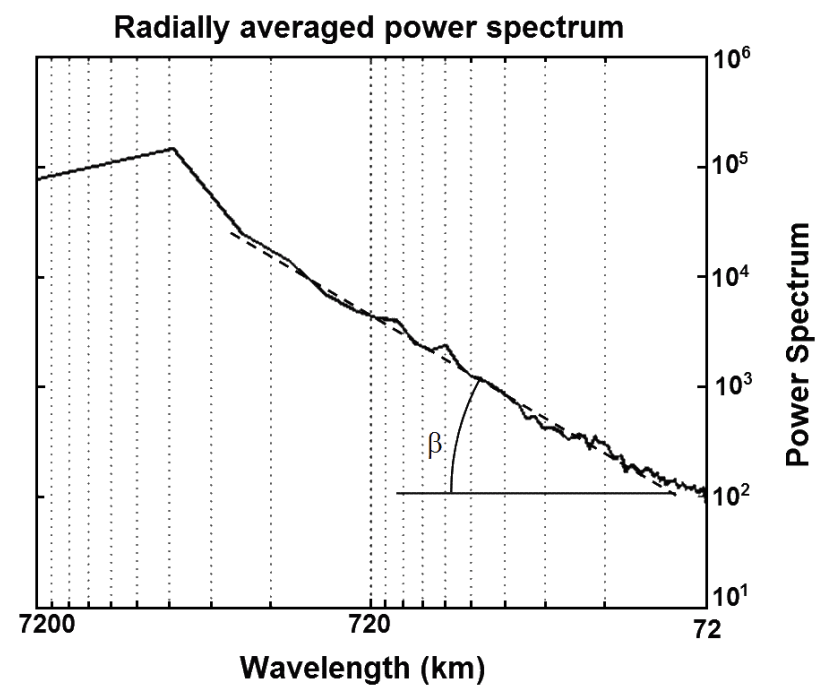

Figure 3: Typical aspect of a graph for the radially-averaged power spectrum $S_{\omega}$ versus wavelength $(\lambda=1 / \omega)$ in a cortical bone sample (adapted from [Sánchez-Molina et al., 2013]).

For investigating the relation between FD and the mechanical properties, we looked at the distribution of regions with high or low mineral density. Indeed, cortical bone presents a very complex and irregular distribution of regions of high and low mineral density. This irregular distribution can be characterized by the FD (a non-dimensional number). According to literature, the mechanical properties (tensile strength and Young's modulus) are related to density. We expect to find correlations between mechanical properties and our measure of the distribution of density, namely, FD. In some previous research studying local differences in density and using fractal analysis, it was shown that fractal analysis led to a relation between the Young's modulus and FD given by [Sánchez-Molina et al., 2013]:

$$
E=E_{0} s^{-\mathrm{FD}}
$$

where $s$ is a scale parameter, and $E_{0}$ is similar to the Young's modulus of pure hydroxyapatite crystals (both $s$ and $E_{0}$ can be estimated by regression analysis). For the tensile strength $\left(\sigma_{\max }\right)$ a similar relation is conjectured (given that tensile strength and Young's modulus are positively correlated):

$$
\sigma_{\max }=\sigma_{0} \hat{s}^{-\mathrm{FD}}
$$

Previous studies have shown that it is possible to relate mechanical properties to the proportion of pores (as measured by FD) or local density (by means of an exponent related to FD) [Sánchez-Molina et al., 2013, Kerrigan et al., 2014]. 


\subsection{Data processing and Distribution of parameters}

The strain of the coupon specimens was computed by DIC: A script in MAT$\mathrm{LAB}^{\circledR}$ was used to process all the frames of the video of the mechanical tests, performing a motion tracking on digital videos. The script allows the creation of a grid of points and the tracking of each of these points in the video record of the tensile test. Thus, with the displacement obtained by means of motion tracking, strain can be determined.

The influence of the anthropometric variables (sex, age, BMI), and the FD on the mechanical properties of the coupon specimens was analyzed using both linear and nonlinear regression analysis. The aim of using linear analysis is to assess if there is a significant effect of FD in mechanical properties (by means of $p$-values of regression analysis). In order to explore whether the relationship between FD and mechanical properties could be significant, it was estimated that a number of specimens $n>50$ would be needed. Then, the nonlinear regression analysis was used, in a second stage, to investigate if the predicted relations (3) and (4) are held by the data in our sample.

A great amount of variability in mechanical properties was observed among individuals of different ages. In order to provide confidence intervals in many practical applications, it is important to know the observed probability distribution for the different mechanical properties. Thus, a fitting to a distribution for each mechanical property and for FD was performed by using the XL-STAT ${ }^{\circledR}$ statistical package by Addinsoft (Paris, France). In principle, it seems that a large number of probability distribution could represent the data. Indeed, we have checked the most commonly used distributions, but there is empirical and theoretical evidence for preferring a specific type of distribution. Firstly, for the maximum strength, many studies have found that Weibull and similar distributions provide an adequate model for the distribution of ultimate strength in different materials [Manzato et al., 2012, Sánchez-Molina et al., 2015]. Secondly, there are theoretical reasons for the occurrence of Weibull distributions. Actually, the fact of the occurrence of Weibull distributions is well understood and it is related to the main result in Extreme Value Theory (EVT), known as Fisher-Tippett-Gnedenko theorem. Given that the macroscopic breaking usually begins in the weakest point of all the regions with the same stress, it is expected the failure to be dominated by the lowest strength points in the high stress region. Therefore, some kind of extreme lower value is implied and, as every statistician knows, these situations are related to the probability limits of EVT. Indeed, the Fisher-Tippett-Gnedenko theorem applied to the stress entails that the distribution of the ultimate strength is a Generalized Extreme Value distribution (GEV distribution). The Weibull distribution is just a particular case of GEV distribution.

So, although a good number of distributions need to be investigated and tested to check suitability, it is expected to find some kind of GEV distribution, as the previous empirical results and the theoretical evidence suggest (at least 
for the tensile strength and, likely, other mechanical properties). The cumulative probability distribution of GEV type is given by:

$$
F(x ; \beta, \bar{\mu}, \kappa)=\exp \left\{-\left[1-\kappa\left(\frac{x-\bar{\mu}}{\beta}\right)\right]^{1 / \kappa}\right\}
$$

where $F(\cdot)$ represents the cumulative distribution function, $x$ is the value of the mechanical property under study, and $\bar{\mu}, k$ and $\beta$ represent the parameters of the GEV distribution (when $\kappa \rightarrow 0$ the equation (5) reduces to the Gumbel distribution). Moreover, the cases with $\kappa>0$ represents a Weibull distribution and for $\kappa<0$ it results a Fréchet distribution.

\section{Results}

For all the tested specimens $(n=66)$, the tensile strength $\left(\sigma_{\max }\right)$, yield strength $\left(\sigma_{0.2}\right)$, maximum strain $\left(\varepsilon_{\max }\right)$, Young's modulus $(E)$, and work to fracture $(W)$ were determined experimentally. Moreover, for these coupon specimens the fractal dimension (FD) was obtained as described in section 2.2. A comparison with other studies is offered Table 1.

Table 1: Average and standard deviation (SD) values for mechanical and geometrical properties, minimum value (Min Value) and maximum value (Max Value) of the observed range in the sample, and comparison with other authors in the literature.

\begin{tabular}{c|cc|cc|ccc}
\hline Property & $\begin{array}{c}\text { Average } \\
\text { Value }\end{array}$ & $\begin{array}{c}\text { Standard } \\
\text { Deviation }\end{array}$ & $\begin{array}{c}\text { Min } \\
\text { Value }\end{array}$ & $\begin{array}{c}\text { Max } \\
\text { Value }\end{array}$ & $\begin{array}{c}\text { Kemper } \\
\text { et al. }\end{array}$ & $\begin{array}{c}\text { Subit } \\
\text { et al. }\end{array}$ & $\begin{array}{c}\text { Katzenberger } \\
\text { et al. }\end{array}$ \\
\hline$\sigma_{\max }[\mathrm{MPa}]$ & 129 & 37.5 & 42.8 & 195 & 124.2 & $112.1 \pm 24.5$ & $53 \div 149$ \\
$\sigma_{0.2}[\mathrm{MPa}]$ & 116 & 26.8 & 36.0 & 164 & 93.9 & - & $38.9 \div 97.2$ \\
$E[\mathrm{GPa}]$ & 16.0 & 3.85 & 7.37 & 27.2 & 13.9 & $13.5 \pm 2.6$ & $11.1 \div 24.4$ \\
\hline$\varepsilon_{\max }$ & 0.0173 & 0.0105 & 0.0036 & 0.0546 & 0.0271 & $0.0106 \pm 0.0029$ & $0.011 \div 0.053$ \\
$\mathrm{FD}$ & 1.740 & 0.058 & 1.536 & 1.873 & - & - & - \\
\hline$W_{\text {fract }}\left[\mathrm{kJ} / \mathrm{m}^{3}\right]$ & 1.80 & 1.68 & 0.118 & 8.69 & - & - & $0.78 \div 4.28$ \\
\hline
\end{tabular}

\subsection{Influence of anthropometric variables and fractal di- mension}

This section uses both linear regression analysis and nonlinear regression analysis to investigate the predictive power of FD and anthropometric variables (i.e. age, sex, and BMI) for the value of the mechanical properties (i.e. Young's modulus, tensile strength, yield strength, maximum strain, and work to fracture).

In the first stage, crude linear regressions were used to investigate significant effects of FD and other anthropometric variables on the mechanical properties. Also, the correlations among variables were investigated (the significant variables and the corresponding $p$-values are shown in Table 2). A significant influence of the FD was found on the tensile strength $(p<0.004)$, the yield strength $(p=0.004)$, and the Young's modulus $(p<0.029)$. Moreover, it can be 
observed that in all cases the inclusion of FD as a regression variable involves an increase in the percentage of explained variability (see Table 2 and Figure 4). These statistical facts suggest being worth considering FD as a descriptor of micro-structural factors.

Table 2: Statistical influence of anthropometric variables and fractal dimension on mechanical properties.

\begin{tabular}{cccccccc}
\hline & \multicolumn{3}{c}{ Values considering FD } & \multicolumn{3}{c}{ Values without FD } \\
\cline { 2 - 7 } & $\alpha_{i}$ & $\beta i$ & $\gamma_{i}$ & $\mathrm{r}$ & $\alpha_{i}$ & $\beta i$ & $r$ \\
\hline$\sigma_{\max }=\alpha_{2}+\beta_{2}$ age $+\gamma_{2} \mathrm{FD}$ & $\begin{array}{c}490.4 \\
(<0.0001)\end{array}$ & $\begin{array}{c}-0.813 \\
(<0.0001)\end{array}$ & $\begin{array}{c}-179.4 \\
(0.004)\end{array}$ & 0.56 & $\begin{array}{c}180.4 \\
(<0.0001)\end{array}$ & $\begin{array}{c}-0.85 \\
(<0.0001)\end{array}$ & 0.46 \\
\hline$\sigma_{0.2}=\alpha_{3}+\beta_{3}$ age $+\gamma_{3} \mathrm{FD}$ & $\begin{array}{c}385.8 \\
(<0.0001)\end{array}$ & $\begin{array}{c}-0.437 \\
(0.005)\end{array}$ & $\begin{array}{c}-142.6 \\
(0.004)\end{array}$ & 0.48 & $\begin{array}{c}139.5 \\
(<0.0001)\end{array}$ & $\begin{array}{c}-0.468 \\
(0.004)\end{array}$ & 0.34 \\
\hline$E=\alpha_{1}+\beta_{1}$ age $+\gamma_{1} \mathrm{FD} \cdot$ age & $\begin{array}{c}16.16 \\
(<0.0001)\end{array}$ & $\begin{array}{c}0.552 \\
(0.032)\end{array}$ & $\begin{array}{c}-0.318 \\
(0.029)\end{array}$ & 0.28 & $\begin{array}{c}16.55 \\
(<0.0001)\end{array}$ & -0.01 & 0.05 \\
\hline$e_{\max }=\alpha_{4}+\beta_{4}$ age $+\gamma_{4} \mathrm{FD} \cdot$ age & $\begin{array}{c}2.893 \\
(<0.0001)\end{array}$ & - & $\begin{array}{c}-0.011 \\
(0.004)\end{array}$ & 0.34 & $\begin{array}{c}2.889 \\
(<0.0001)\end{array}$ & $\begin{array}{c}-0.019 \\
(0.005)\end{array}$ & 0.34 \\
\hline
\end{tabular}

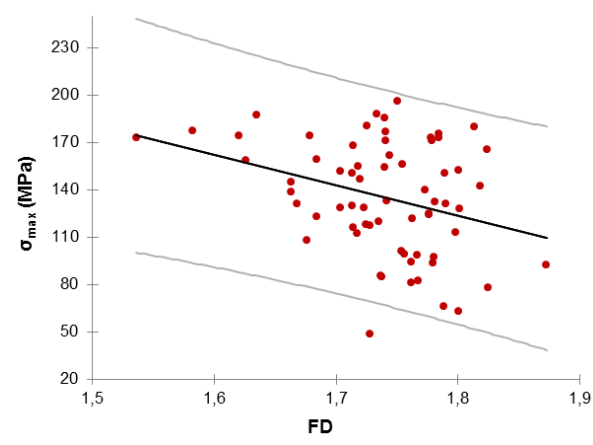

(a) Tensile strength

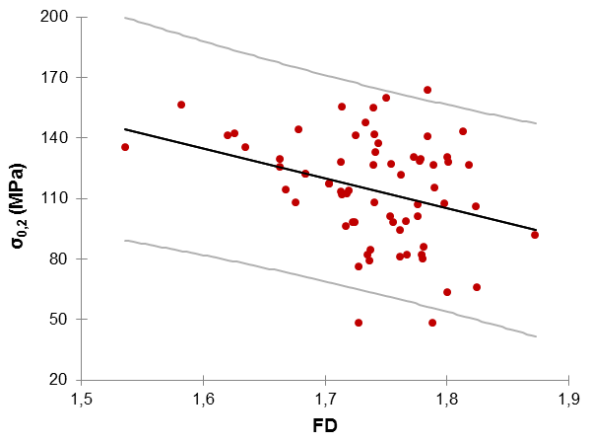

(b) Yield strength

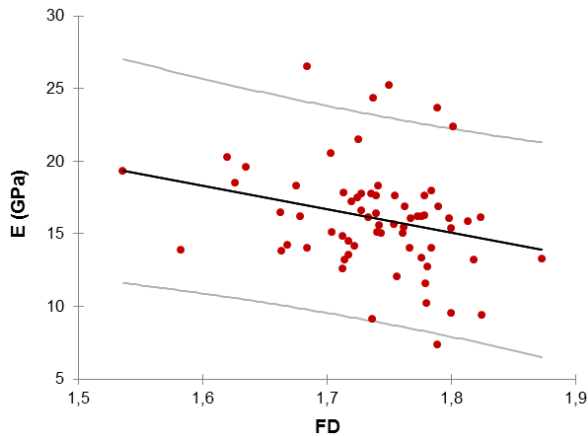

(c) Strain

Figure 4: Scattering plot of the mechanical properties versus Fractal Dimension (FD): (a) tensile strength, (b) yield strength, (c) Young's modulus. All the plots show a diminution in the value of the mechanical properties when FD increases. 
In the second stage, nonlinear regression analysis was used to investigate if the relation between the mechanical properties obtained from the experimental procedure and the FD computed from $\mathrm{CT}$ images follows the proposed powerlaw relations (3) and (4), as it was previously suggested in the literature. In particular, for the Young's modulus, we selected the nonlinear regression form:

$$
E=E_{0}(\text { age }, \mathrm{FD}) s_{1}^{-\mathrm{FD}}, \quad E_{0}(\text { age }, \mathrm{FD})=\alpha_{1}+\beta_{1} \text { age }+\gamma_{1} \mathrm{FD}
$$

So, the non-linear regression was used to estimate the regression coefficients $s_{1}, \alpha_{i}, \beta_{i}, \gamma_{i}$. According to equation (4) a similar regression form was selected:

$$
\sigma_{\max }=\sigma_{0}(\text { age }, \mathrm{FD}) s_{2}^{-\mathrm{FD}}, \quad \sigma_{0}(\text { age }, \mathrm{FD})=\alpha_{2}+\beta_{2} \text { age }+\gamma_{2} \mathrm{FD}
$$

This non-linear analysis showed a remarkable increase in the $r^{2}$ values $\left(r^{2}\right.$ increases from 0.12 to 0.50 depending on the mechanical property analyzed). The results obtained in this nonlinear analysis are summarized in Table 3.

\begin{tabular}{|c|c|c|c|c|c|}
\hline & $s_{i}$ & $\alpha_{i}$ & $\beta_{i}$ & $\gamma_{i}$ & $r$ \\
\hline$E=E_{0}($ age, $\mathrm{FD}) s_{1}^{-\mathrm{FD}}$ & $\begin{array}{c}3.031 \\
(0.023)\end{array}$ & $\begin{array}{c}1.874 \\
(<0.0001)\end{array}$ & - & $\begin{array}{c}1.077 \\
(<0.0001)\end{array}$ & 0.51 \\
\hline$\sigma_{\max }=\sigma_{0}($ age, $\mathrm{FD}) s_{2}^{-\mathrm{FD}}$ & $\begin{array}{c}5.103 \\
(0.004)\end{array}$ & $\begin{array}{c}54.94 \\
(<0.0001)\end{array}$ & $\begin{array}{c}-0.046 \\
(<0.0001)\end{array}$ & $\begin{array}{c}-25,59 \\
(<0.0001)\end{array}$ & 0.74 \\
\hline$\sigma_{0.2}=\overline{\sigma_{0}}($ age, $\mathrm{FD})$ & $\begin{array}{c}4.349 \\
(0.003)\end{array}$ & $\begin{array}{c}56.09 \\
(<0.0001)\end{array}$ & $\begin{array}{l}-0.033 \\
(0.005)\end{array}$ & $\begin{array}{c}-26.07 \\
(<0.0001)\end{array}$ & 0.71 \\
\hline
\end{tabular}

Table 3: Relation between anthropometric variables and FD, using nonlinear regression.

Table 3 shows that the mechanical properties diminish with age $\left(\beta_{2}, \beta_{3}, \beta_{4}<\right.$ $0)$. In addition, an increase in FD produces a decrease in the mechanical properties (even when there are second-order corrections).

\subsection{Distribution fittings of mechanical properties}

For each mechanical property, a high variability has been observed. Thus, to describe the distribution of the obtained values, a fitting to a distribution was performed for each property. The results of these fittings are shown in Figure 5 and Table 4. Tensile strength, yield strength, Young's modulus, maximum strain, and FD show a good fitting to a GEV-type distribution (Weibull or Gumbel). On the other hand, work to fracture fits better to an exponential distribution, with cumulative distribution function of the form:

$$
F(x ; \lambda)=1-\mathrm{e}^{-\lambda x}
$$

GEV-type distributions are defined by three parameters $(\beta, \bar{\mu}, \kappa)$, and are represented by a cumulative distribution function given by equation (5). Notice that, as described above, the shape parameter $\kappa$ defines the type of the GEV 


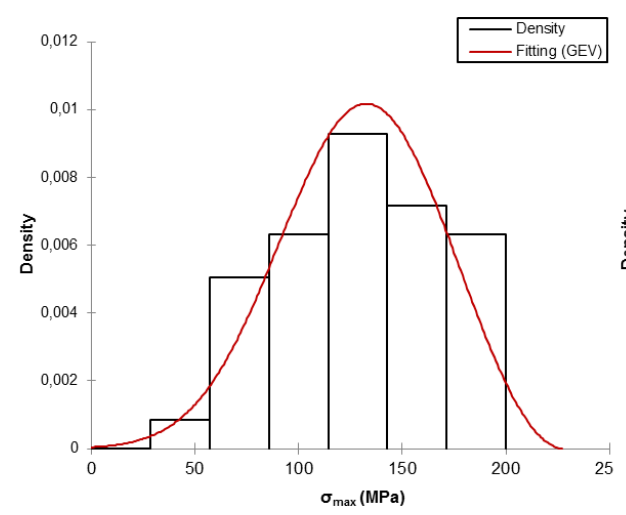

(a) Tensile strength

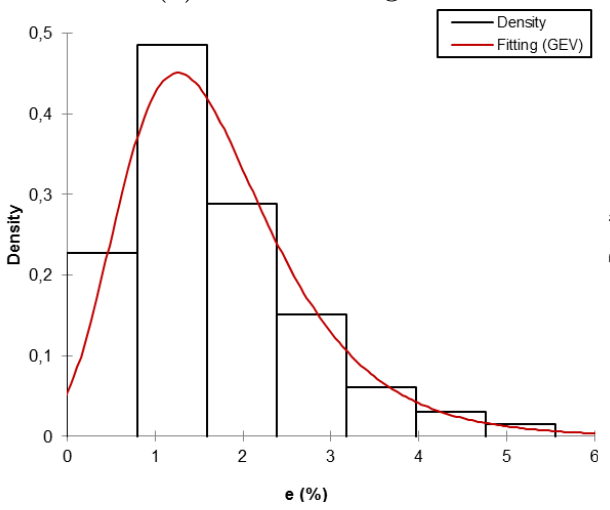

(c) Strain

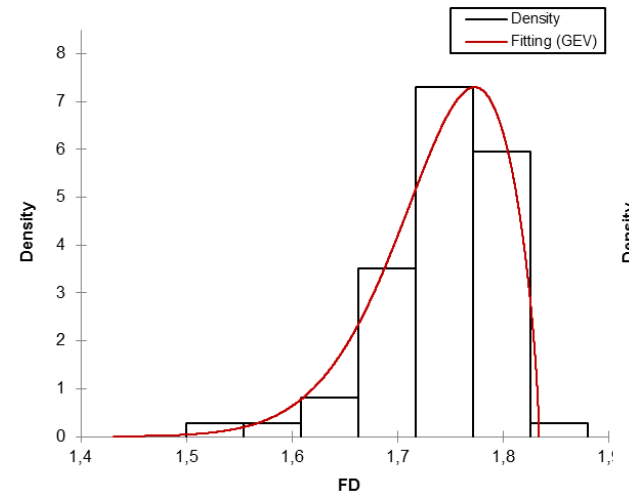

(e) Fractal Dimension

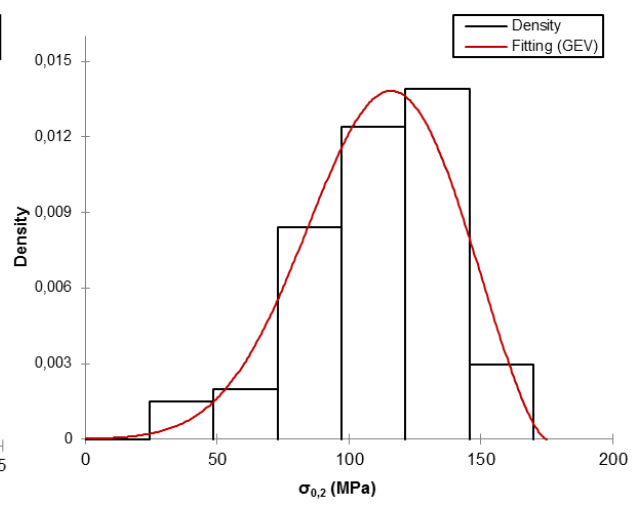

(b) Yield strength

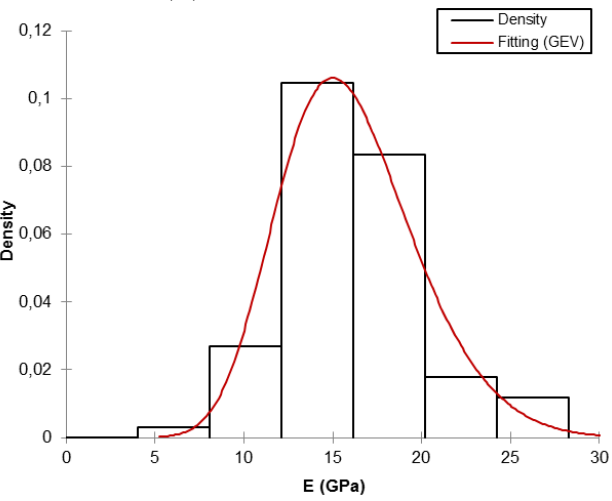

(d) Young's Modulus

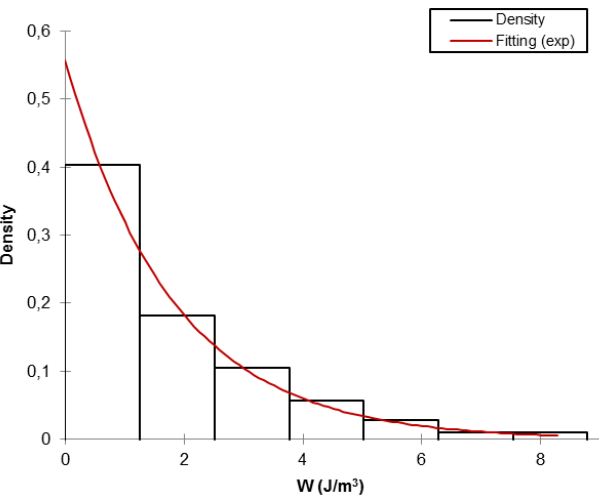

(f) Work to fracture

Figure 5: Distribution fitting of mechanical properties determined: tensile strength (a), yield strength (b), maximum strain (c), Young modulus (d) and FD (e) fit to a Gumbel distribution, strain (b) fit to a Weibull distribution and work to fracture (f) to an exponential distribution. 
Table 4: Parameter values of the distribution fittings of mechanical properties and fractal dimension determined.

\begin{tabular}{|c|c|c|c|c|c|}
\hline \multirow{2}{*}{ Distribution } & \multirow{2}{*}{ Property } & \multicolumn{3}{|c|}{ Parameter } & \multirow{2}{*}{$\begin{array}{c}p \text {-value } \\
\text { (Kolmogorov-Smirnov) }\end{array}$} \\
\hline & & $\kappa$ & $\beta$ & $\bar{\mu}$ & \\
\hline \multirow{5}{*}{ GEV } & $\sigma_{\max }$ & 0.352 & 38.81 & 117.03 & 0.834 \\
\hline & $\sigma_{0.2}$ & 0.403 & 29.25 & 102.23 & 0.947 \\
\hline & $E$ & 0.147 & 3.51 & 14.44 & 0.373 \\
\hline & $\varepsilon_{\max }$ & 0 & 0.816 & 1.26 & 0.691 \\
\hline & FD & 0.601 & 0.064 & 1.73 & 0.740 \\
\hline \multicolumn{5}{|c|}{$\lambda$} & \\
\hline Exponential & $W_{\text {fract }}$ & 0.556 & & & 0.484 \\
\hline
\end{tabular}

distribution. The average maximum strain $\varepsilon_{\max }$ was $0.0173 \pm 0.0105$, and for this property the results showed a good fitting to a Gumbel distribution. For all the other magnitudes (tensile strength $\sigma_{\max }$, yield strength $\sigma_{0.2}$, Young's modulus $E$, and fractal dimension FD), we have found that a Weibull distribution adequately represents the data. For all these mechanical properties, some additional details of their range of variation are given in Table 1.

\section{Discussion}

In this study, the mechanical properties of human rib cortical bone tissue have been obtained from a relatively innovative tensile test method performed with dog-shape specimens (or coupons). The procedure isolates intrinsic mechanical properties from the extrinsic geometrical component of the ribs. The measured values for tensile strength, yield strength, maximum strain, and Young's modulus are consistent with those determined in other studies [Sánchez-Molina et al., 2013, Katzenberger et al., 2020].

The data show that there is a highly significant effect of FD in the mechanical properties. This finding agrees with previous studies in cortical and trabecular bone [Huh et al., 2006, Sánchez-Molina et al., 2013, Kerrigan et al., 2014]. The results confirm that FD is a good measure of porosity and/or regions of lower density, as it was previously suggested by other authors studying morphological factors to predict osteoporosis [Tosoni et al., 2006, Lespessailles et al., 2008]. Two remarkable characteristics of this study are the relatively high number of different individuals sampled, and the avoiding of classical box-counting method for estimating the $\mathrm{FD}$, given the drawbacks that such a procedure presents [Camastra, 2003], even though some recent improvements of box-counting-type methods have been proposed [Raghavendra and Dutt, 2010].

We have corroborated that predictions of fractal analysis for micro-structure 
reflected in Equations (3) and (4) provide a much better fitting than simple linear regressions. In addition, our data provide an interesting link between FD and a great amount of scientific literature focused on the relationship of BMD and mechanical properties. A good survey of the results in this literature is reviewed in [Helgason et al., 2008]. In essence, many studies have shown that for the Young's modulus $(E)$ and the density $(\rho)$ one obtains a relation $E=C_{1} \rho^{\alpha}$ with $2.3 \leq \alpha \leq 3.1$. On the other hand, fractal analysis suggests that $\rho=C_{2} \bar{s}^{\mathrm{FD}}$ [Sánchez-Molina et al., 2013], and these equations entails a relation $E=\left(C_{1} C_{2}^{\alpha}\right)\left(\bar{s}^{\alpha}\right)^{-\mathrm{FD}}=E_{0} s^{-\mathrm{FD}}$, for which we have presented solid additional evidence in this paper by using a high number of specimens, providing sufficient statistical power.

Finally, we have found evidence that the variation in mechanical properties can be adequately represented by Weibull/GEV-type distributions. The occurrence of such distributions is explained because bone is a brittle material with many similarities with ceramic materials, for which a weakest link argument leads to GEV distributions. For this reason, it is expected to find Weibull distributions for most mechanical properties as the pioneering work of Weibull suggests. The Weibull theory was formulated in 1939, and it is a statistical theory of failure based on weakest link assumption [Weibull, 1939]. Being the well-known Extreme Value Theory a generalization of the Weibull theory, the application of this theory to a brittle material with porosity and/or microstructure leads to a GEV distribution [Vořechovský \& Sadílek, 2008].

Nevertheless, some limitations of this study need to be pointed out. For instance, only quasi-static tests were performed, and the data do not provide any information about viscoelastic effects or degradation/previous damage effects of the type considered [Jepsen and Davy, 1997, Joo em et al., 2007]. Possibly, the previous history of the bone tissue or previous traumas could affect the applicability of Weibull theory. Although some data from the autopsy are anonymously provided, it is not possible to discard that some uncontrollable factors affect the distributions found for $\sigma_{\max }, \sigma_{0.2}$, and $\varepsilon_{\max }$. So, further work is needed in order to determine the effect of deterioration or previous damage in the final strength of cortical bone.

\section{Conclusion}

A significant effect of FD on most mechanical properties of human rib cortical bone was found. This result is of high importance because it opens the possibility to use FD (measurable by a noninvasive DIC) to assess mechanical properties of pathologically affected bone tissue in a noninvasive technique. Indeed, FD seems a strong predictor of occurrence of porosity or lower density in cortical bone (the main mechanical properties decrease when FD increases), and so diminished tensile strength.

In addition, the influence of age on the mechanical properties was observed (except for the Young's modulus). In all cases, an increase in age leads to a 
reduction of the mechanical properties. The influence of BMI was only observed on maximum strain: An increase of the BMI implies a decrease of strain capacity. The biological sex only showed significant effect on tensile strength, i.e., the bone strength in females being lower than in males. Most mechanical properties are distributed according to a GEV distribution. This allows defining confidence intervals for specific practical applications.

\section{From DECLARATION FORM:}

(1) The authors declare no conflict of interest, (2) the authors conducted this research without the help of any source of funding, and (3) this study does not involve human subjects [the samples used are from PMHS and were collected only after a permission from the relatives, according to ethical directives of IMLCFC].

\section{References}

[Camastra, 2003] Camastra, F. (2003): "Data dimensionality estimation methods: a survey". Pattern recognition, 36(12), pp. 2945-2954.

[Dougherty \& Henebry, 2001] Dougherty, G., \& Henebry, G. M. (2001): "Fractal signature and lacunarity in the measurement of the texture of trabecular bone in clinical CT images", Medical Engineering \& Physics, 23(6), pp. 369-380.

[Haire et al., 1998] Haire, T. J., Hodgskinson, R., Ganney, P. S., \& Langton, C. M. (1998): "A comparison of porosity, fabric and fractal dimension as predictors of the Young's modulus of equine cancellous bone", Medical engineering \& physics, 20(8), pp. 588-593.

[Harrar \& Jennane, 2015] Harrar, K., \& Jennane, R. (2015): "Trabecular Texture Analysis Using Fractal Metrics for Bone Fragility Assessment", International Journal of Medical Health, Biomedical, Bioengineering and Pharmaceutical Engineering, 9(69), pp. 683-688.

[Helgason et al., 2008] Helgason, B., Perilli, E., Schileo, E., Taddei, F., Brynjólfsson, S., \& Viceconti, M. (2008): "Mathematical relationships between bone density and mechanical properties: a literature review", Clinical biomechanics, 23(2), pp. 135-146.

[Huh et al., 2006] Huh, K.H., Yi, W.J., Jeon, I.S., Heo, M.S., Lee, S.S., Choi, S.C., Lee, J.I., \& Lee, Y.K. (2006): "Relationship between twodimensional and three-dimensional bone architecture in predicting the mechanical strength of the pig mandible", Oral Surgery, Oral Medicine, Oral Pathology, Oral Radiology, and Endodontology, 101(3), pp. 363-373.

[Jepsen \& Davy, 1997] Jepsen, K.J., \& Davy, D.T. (1997): "Comparison of damage accumulation measures in human cortical bone". Journal of Biomechanics, 30(9), pp. 891-894. 
[Joo et al., 2007] Joo, W., Jepsen, K.J., \& Davy, D. T. (2007): "The effect of recovery time and test conditions on viscoelastic measures of tensile damage in cortical bone". Journal of Biomechanics, 40(12), pp. 2731-2737.

[Katzenberger et al., 2020] 20] Katzenberger, M. J., Albert, D. L., Agnew, A. M., \& Kemper, A. R. (2020): "Effects of sex, age, and two loading rates on the tensile material properties of human rib cortical bone", Journal of the mechanical behavior of biomedical materials, 102, 103410.

[Kemper et al., 2005] Kemper, A.R., McNally, C., Kennedy, E.A., Manoogian, S.J., Ratch, A.L., ng, T.P, \& Matsuoka, F. (2015): "Material properties of human rib cortical bone from dynamic tension coupon testing", Stapp Car Crash J, 49(11), pp. 199-230.

[Kerrigan et al., 2014] Kerrigan, J. R., Sánchez-Molina, D., Neggers, J., Arregui-Dalmases, C., Velázquez-Ameijide, J., \& Crandall, J. R. (2014): "Indentation response of human patella with elastic modulus correlation to localized fractal dimension and bone mineral density". Journal of the mechanical behavior of biomedical materials, 33, pp. 99-108.

[Lespessailles et al., 1998] Lespessailles, E., Jullien, A., Eynard, E., Harba, R., Jacquet, G., Ildefonse, J. P., ...\& Benhamou, C. L. (1998): "Biomechanical properties of human os calcanei: relationships with bone density and fractal evaluation of bone microarchitecture", Journal of Biomechanics, 31(9), pp. 817-824.

[Lespessailles et al., 2008] Lespessailles, E., Gadois, C., Kousignian, I., Neveu, J.P., Fardellone, P., Kolta, S., Roux, C., Do-Huu, J.P., \& Benhamou, C.L. (2008): "Clinical interest of bone texture analysis in osteoporosis: a case control multicenter study". Osteoporosis international, 19(7), pp. 10191028 .

[Lin et al., 1999] Lin, J. C., Grampp, S., Link, T., Kothari, M., Newitt, D. C., Felsenberg, D., \& Majumdar, S. (1999): "Fractal analysis of proximal femur radiographs: correlation with biomechanical properties and bone mineral density". Osteoporosis international, 9(6), pp. 516-524.

[Manzato et al., 2012] Manzato, C., Shekhawat, A., Nukala, P., Alava, M.J., Sethna, J.P., Zapperi, S., (2012): "Fracture strength of disordered media: universality, interactions, and tail asymptotics", Phys. Rev. Lett., 108(6), 065504 .

[Odgaard et al., 1997] Odgaard, A., Kabel, J., van Rietbergen, B., Dalstra, M., \& Huiskes, R. (1997): "Fabric and elastic principal directions of cancellous bone are closely related", Journal of Biomechanics, 30(5), pp. 487-495.

[Raghavendra \& Dutt, 2010] Raghavendra, B.S., \& Dutt, N. D. (2010): "Computing fractal dimension of signals using multiresolution box-counting method", International Journal of Information and Mathematical Sciences, 6 (1), pp. 50-65. 
[Rho et al., 1997] Rho, J.Y., Flaitz, D., Swarnakar, V., \& Acharya, R.S. (1997): "The characterization of broadband ultrasound attenuation and fractal analysis by biomechanical properties", Bone, 20(5), pp. 497-504.

[Sánchez \& Uzcátegui, 2011] Sánchez, I., Uzcátegui, G. (2011): "Fractals in dentistry", Journal of dentistry, 39 (4), pp. 273-292.

[Sánchez-Molina et al., 2013] Sánchez-Molina, D., Velázquez-Ameijide, J., Quintana, V., Arregui-Dalmases, C., Crandall, J.R., Subit, D., Kerrigan, J.R. (2013): "Fractal dimension and mechanical properties of human cortical bone", Medical engineering $\& 3$ physics, 35 (5), pp. 576-582.

[Sánchez-Molina et al., 2015] Sánchez-Molina, D., Martínez-González, E., Velázquez-Ameijide, J., Llumà, J., Soria, M.R., \& Arregui-Dalmases, C. (2015): "A stochastic model for soft tissue failure using acoustic emission data", Journal of the mechanical behavior of biomedical materials, 51, pp. $328-336$.

[Sindeaux et al., 2014] Sindeaux, R., de Souza Figueiredo, P. T., de Melo, N. S., Guimarães, A. T. B., Lazarte, L., Pereira, F. B., de Paula, A.P., \& Leite, A. F. (2014): "Fractal dimension and mandibular cortical width in normal and osteoporotic men and women", Maturitas, 77(2), pp. 142-148.

[Subit et al., 2011] [Subit, D., de Dios, E. D. P., Velázquez-Ameijide, J., Arregui-Dalmases, \& C., Crandall, J. (2011): "Tensile material properties of human rib cortical bone under quasi-static and dynamic failure loading and influence of the bone microstucture on failure characteristics", arXiv preprint, arXiv:1108.0390.

[Tosoni et al., 2006] Tosoni, G.M., Lurie, A.G., Cowan, A.E., \& Burleson, J. A. (2006): "Pixel intensity and fractal analyses: detecting osteoporosis in perimenopausal and postmenopausal women by using digital panoramic images.", Oral Surgery, Oral Medicine, Oral Pathology, Oral Radiology, and Endodontology, 102(2), pp. 235-241.

[Töyräs et al., 1999] Töyräs, J., Kröger, H., \& Jurvelin, J.S. (1999): "Bone properties as estimated by mineral density, ultrasound attenuation, and velocity.", Bone, 25(6), pp.725-731.

[Vořechovský \& Sadílek, 2008] Vořechovský, M., \& Sadílek, V. (2008): "Computational modeling of size effects in concrete specimens under uniaxial tension", International Journal of Fracture, 154(1-2), pp. 27-49.

[Weibull, 1939] Weibull, W. 1939: "A statistical theory of the strength of material", Ingeniors Vetenskaps Akademiens, Stockholm, 151.

[Yin et al., 2015] Yin, D., Chen, B., Ye, W., Gou, J., \& Fan, J. (2015): "Mechanical test and fractal analysis on anisotropic fracture of cortical bone", Applied Surface Science, 357, pp. 2063-2068. 
[Zioupos et al., 2006] Zioupos, P., Kaffy, C., \& Currey, J. D. (2006): "Tissue heterogeneity, composite architecture and fractal dimension effects in the fracture of ageing human bone", International Journal of Fracture, 139(34), pp. 407-424. 